\title{
Centralised databases as a primer for research
}

An all-Australian research effort leads this Australian Supplement to The Journal of Laryngology \& Otology. Prof Cervin's group at Brisbane conduct a terrific piece of research utilising current Cancer Research databases. ${ }^{1}$ Such research demonstrates the untapped potential of health databases that are available to researchers in Australia. We are fortunate to have a socialised healthcare system, but all too often overlook the benefits of such for research. Government and quasi-government groups collate enormous amounts of data for the purposes of auditing the healthcare system and providing future policy guidance. The Australian Institute of Health and Welfare $^{2}$ and the Health section of the Australian Bureau of Statistics ${ }^{3}$ are two good electronic portals to initiate future research. Broader Medicare and pharmaceutical benefit scheme data are also available. The authors' finding of a steady increase in mucosal melanoma incidence is unlikely to be a result of reporting alone, and such data act as a primer for future research.

Piezoelectric technology has made its impact in otolaryngology, from otological surgery, to skull base and even rhinoplasty work. The review from Meller and Havas is a great example of how to avoid a narrative review, and produce a quality review that is both comprehensive and unbiased. ${ }^{4}$ The work follows the theme of compliance with the Preferred Reporting Items for Systematic Reviews and Meta-Analyses ('PRISMA'), ${ }^{5}$ which is used as a template for their systematic review. The Journal is always very open to such quality review work.

Finally, Prof Vijayasekaran's group produce some original data on post-tonsillectomy bleeding. ${ }^{6}$ It is no surprise here that experience more than technology leads to better outcomes. The literature is replete with tonsillectomy trials. These are mainly industry funded, and usually aimed at providing evidence for a new technique or technology. Very little of this actually changes clinical practice. Independent research such as that by Hinton-Bayre and colleagues is required to ensure that the research we do is both important and clinically relevant.

With the recent scientific meeting of the Australian Society of Otolaryngology Head and Neck Surgeons, in Adelaide, I encourage all researchers to consider The Journal for publication of their work.

\section{RICHARD HARVEY \\ Editor \\ Australian Supplement of The Journal of Laryngology \& Otology}

References

1 Youssef D, Vasani S, Marquess J, Cervin A. Rising incidence of head and neck mucosal melanoma in Australia. $J$ Laryngol Otol 2017;131(suppl 2):S25-8

2 Australian Institute of Health and Welfare. In: http://www.aihw. gov.au/home/ [24 April 2017]

3 Australian Bureau of Statistics. In: http://www.abs.gov.au/ browse?opendocument\&ref=topBar [24 April 2017]

4 Meller C, Havas TE. Piezoelectric technology in otolaryngology, and head and neck surgery: a review. J Laryngol Otol 2017; 131(suppl 2):S12-8

5 Preferred Reporting Items for Systematic Reviews and MetaAnalyses. In: http://www.prisma-statement.org/ [24 April 2017]

6 Hinton-Bayre AD, Noonan K, Ling S, Vijayasekaran S. Experience is more important than technology in paediatric post-tonsillectomy bleeding. J Laryngol Otol 2017;131(suppl 2):S35-40 